\title{
Mathematical models for tumours with cancer stem cells
}

\section{Luca Meacci \& Mario Primicerio}

\section{Computational and Applied Mathematics}

\section{ISSN 0101-8205}

Volume 37

Number 5

Comp. Appl. Math. (2018) 37:6544-6559 DOI 10.1007/s40314-018-0707-2
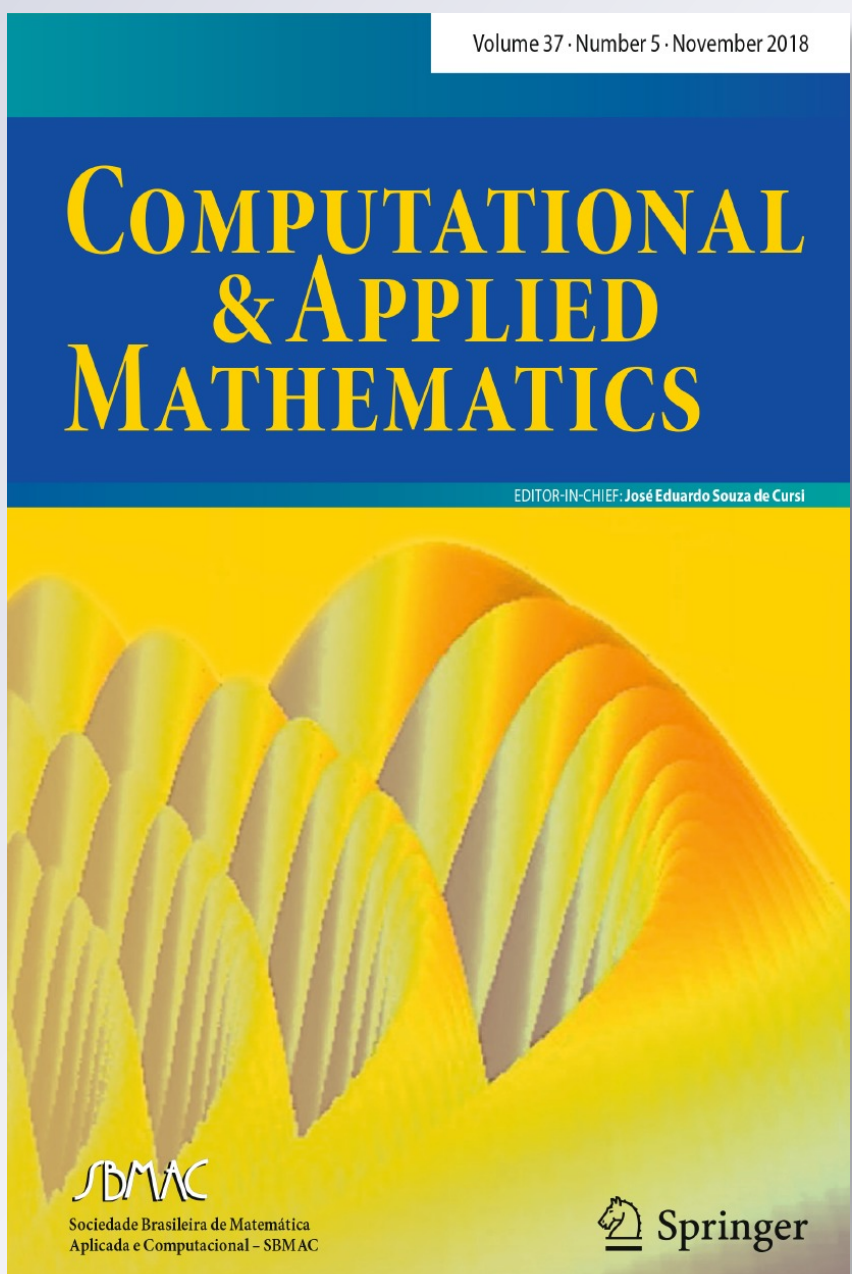

Sociedade Brasileira de Matemática

Springer 
Your article is protected by copyright and all rights are held exclusively by SBMAC Sociedade Brasileira de Matemática Aplicada e Computacional. This e-offprint is for personal use only and shall not be self-archived in electronic repositories. If you wish to self-archive your article, please use the accepted manuscript version for posting on your own website. You may further deposit the accepted manuscript version in any repository, provided it is only made publicly available 12 months after official publication or later and provided acknowledgement is given to the original source of publication and a link is inserted to the published article on Springer's website. The link must be accompanied by the following text: "The final publication is available at link.springer.com". 


\title{
Mathematical models for tumours with cancer stem cells
}

\author{
Luca Meacci $^{1}$ (D) Mario Primicerio ${ }^{2,3}$
}

Received: 21 July 2017 / Revised: 11 August 2018 / Accepted: 24 August 2018 / Published online: 30 August 2018 (c) SBMAC - Sociedade Brasileira de Matemática Aplicada e Computacional 2018

\begin{abstract}
The paper deals with mathematical modelling of the growth of tumours characterized by the presence of cancer stem cells (CSC). These cells are assumed to be immortal and multipotent, in the sense that they can generate either CSC or ordinary cancer cells (CC). We present a synthetic review of some models that have been proposed in the literature and we report the development of new original models, to open a complete multi-approach window on modellization of the problem. The mathematical models are presented without technical details, but numerical simulations are displayed that exhibit the so-called "tumour growth paradox" that consists in the fact that tumours with a larger death rate for the CC may grow faster than tumours with a lower death rate for the same class of cells. The occurrence of this paradox has important consequences also on the strategy of treatment of tumours.
\end{abstract}

Keywords Cancer stem cells · Mathematical modeling of tumours · Cellular automata · Ordinary differential equations · Integro-partial differential equations $\cdot$ Reaction-diffusion equations

Mathematics Subject Classification 93A30 $\cdot 92 \mathrm{Cxx} \cdot 37 \mathrm{~B} 15$

\section{Introduction}

In the last few years several papers have been devoted to the construction, discussion and analysis of mathematical models designed to describe of the growth of tumours in presence of cancer stem cells (CSC), see for instance (Betteridge et al. 2006; Borsi et al. 2015; Enderling et al. 2009; Fasano et al. 2016; Ganguly and Puri 2006; Hillen et al. 2013). The heterogeneity

Communicated by Florence Hubert.

Luca Meacci

luca.meacci@usp.br; luca.meacci@gmail.com

Mario Primicerio

mario.primicerio@unifi.it

1 Instituto de Ciências Matemáticas e de Computação, ICMC, Universidade de São Paulo, Avenida Trabalhador Sancarlense, 400, São Carlos, SP CEP 13566-590, Brazil

2 Dipartimento di Matematica "U. Dini”, Università degli Studi di Firenze, Viale Giovanni Battista Morgagni, 67/A, 50134 Firenze, Italy

3 Istituto per le Applicazioni del Calcolo "Mauro Picone", CNR, Via dei Taurini, 19, 00185 Roma, Italy 
of tumour cells in breast cancers was put in evidence in Al-Hajj et al. (2003) where the term tumorigenic cells was introduced; this fact is now widely accepted (see, e.g., Lathia et al. 2015) and CSC have been identified in many cancers, as well in sarcomas and leukemia (see, e.g., Dittmar and Zänker 2013). The relevance of CSC in the progression of these and other tumours and the investigation of their role has been the object of many experimental papers as well as of more theoretically oriented articles, see (Michor 2008) and the bibliography cited therein as well as (Dingli and Michor 2006; Dittmar and Zänker 2013; Kakarala and Wicha 2008; Lathia et al. 2015; Maddalena 2014; Solé et al. 2008). One of the features of tumours with CSC is the so-called tumour-growth-paradox that consists in the fact that tumours with a larger death rate for ordinary (non-stem) cancer cells might grow bigger than tumours with a lower death rate for the same class of cells (Enderling et al. 2009; Hillen et al. 2013). Of course, since the death rate is influenced by medical treatment, understanding the reasons of this paradox can be relevant in the control of the tumours (Gurova and Gudkov 2003; Wodarz and Komarova 2007). In Rodriguez-Brenes et al. (2017) a negative feedback from CC on the proliferation of CSC is incorporated in the system of the ODE modelling the growth rate, and this fact is considered to originate the paradoxical behaviour in some cases of drug treatment. Concerning mathematical models, that are the object of this short review, we have to stress that they are not intended to give quantitative prediction of the progression of cancers, nor to include all possible factors and aspects that influence their growth. They are rather oriented to offer a conceptualization of the phenomenon and to isolate the mechanisms that seem to be crucial for the essential features of the evolution of tumours in presence of CSC. In other words, the models that we present essentially fall in the "descriptive" camp rather than in the "mechanistic" camp, according to the classification of Anderson and Quaranta (2008). Nevertheless, we believe they can offer some suggestions to conceptualize the phenomenon in terms of its essential components. On the other hand, presenting and analyzing various mathematical modelling techniques and the corresponding numerical simulations outlines in particular the model-independence of the occurrence of the tumour growth paradox. In the models that we describe some drastic simplifications are introduced to provide a mathematical scheme such that the properties of its solutions can be investigated and simulated. On the other hand, it is important that the model does not contain a too high number of parameters, whose values would be hardly estimated form experimental data. In other words, as Albert Einstein said, "a model should be as simple as possible... but not simpler". In this paper, we will present, analyze and discuss some mathematical models that have been proposed in this context (see Betteridge et al. 2006; Borsi et al. 2015; Enderling et al. 2009; Fasano et al. 2016; Hillen et al. 2013; Maddalena 2014; Stiehl and Marciniak-Czochra 2012), avoiding technical details and displaying some numerical simulations. In addition, we will present the development of an original model based on cellular automata to provide a comprehensive overview on modelling of the phenomenon. We will see, in particular, in which sense and cases the models exhibit the paradoxical behaviour outlined above and we will mimic the response of tumours to different treatments. We will not describe mathematical models more based on stochastic arguments and/or dealing with the mutation of the normal cells to CSC (Ganguly and Puri 2006; Sun and Komarova 2015).

\section{Basic ingredients of the models}

The models that we will describe contain two basic simplifications. The first simplification consists in assuming that the population of tumour cells consists of just two sub-populations: cancer stem cells (CSC) and ordinary non-stem cells (CC) and that all the cells in each 
subpopulation have the same properties. This implies in particular that it is assumed (and this is the second drastic approximation) that cells have age-independent replicative potential and mortality. More specifically, for CC there is a fixed probability of generating new CC or of undergoing apoptosis, while CSC are assumed to be immortal and to be capable of generating new CSC (symmetrical mitosis) or CC (unsymmetrical mitosis), with a fixed ratio between the two probabilities. Of course these are very strong simplifications because in practice multipotent cells generate various cell lineages and because cells are subject to senescence and their parameters (replication potential, mortality) vary accordingly. In Rodriguez-Brenes et al. (2014) a model is presented in which the cell population is organized in three types of cells: stem cells, progenitors, and differentiated cells. Mutations associated with malignancies have different effects if they take place in the stem cells or downstream. On the other hand, in the models consisting in systems of equations (ODE, integro differential equations, reaction-diffusion equations) the unknown functions represent averaged fractions of CC and CSC and thus the characteristics of each cell (age among them) cannot be taken into account. This is the limit of this class of models that can represent just a conceptual idea of the complex phenomenon. Nevertheless, even with these simplifications, the models we discuss here are sufficiently rich and in particular they are able to describe the tumour paradox adequately. We may add that it could be possible to encompass age-dependence in the models, introducing compartmental models in which the unknown functions represent the fractions of cells belonging to a sequence of different classes of age, as in Werner et al. (2011, 2016), Michor et al. (2005), and Dingli et al. (2007). The "cost" of this approach will be the introduction of a larger number of parameters that have to be fitted with experimental data. In a different context (hematopoietic development) a multi-compartment model has been proposed (see Marciniak-Czochra et al. 2009) to describe the proliferation (and senescence) of different age classes of cells.

An important effect that is included (although in a very idealized way) in all the models that will be described is the "crowding effect" (Betteridge et al. 2006; Borsi et al. 2015; Enderling et al. 2009; Fasano et al. 2016; Hillen et al. 2013). This means that mitosis is supposed to be inhibited when the density of cells in a neighborhood of the would-be mother cell exceeds a threshold value. Models based on the basic concepts of population dynamics, but not taking into account the crowding effect have been proposed starting from Michor et al. (2005) but were unable to explain the tumour growth paradox. In what follows we do not take into account explicitly the movement of cells induced by mutual pushing by effect of mitosis. In some sense, this mechanism is is implicitly included in the mean field approximation (4) because the "crowding" term $1-u-v$ represents the availability of space in the whole region of interest. ${ }^{1}$ According to some authors (e.g., Betteridge et al. 2006; Enderling et al. 2009; Hillen et al. 2013) "pushing" effect can be included in integro-differential models (see Sect. 5) by adding diffusion and this is reflected, in reaction-diffusion models (see Sect. 6), by additive terms in the coefficients of $U_{x x}$ and $V_{x x}$. From the point of view of the mathematical well-posedness and of the qualitative aspects of the numerical simulations this does not make any relevant difference (just the appearance of two more parameters). In case of agent-based models one could possibly consider different probabilities of replication when space is available in a close neighborhood of the mother cell with respect to the case in which the space is available at some distance and some energy has to be spent to displace the neighboring cells. More generally, some papers have taken into account the role if the extra-cellular matrix (ECM) and the mechanical effects induced by tumour growth (Kiel et al.

1 Possibly, this term could be modified by a different function of $u+v$ vanishing when the whole region is filled by tumour cells. 
2007). This is essential when processes of encapsulation are to be described, but we will not include this process in our models.

\section{Agent-based models}

This approach is based on the so-called "cellular automata" (CA). As it is well-known, CA were introduced by John von Neumann (following the idea of Stanislaw Ulam) to study global properties from local processes (Kari 2005) and are currently applied in several contexts (Chopard and Droz 1998). ${ }^{2}$ In the present case we imagine cells as living in a square (or cubic) lattice and we prescribe (probabilistic) rules for their motion, replication or death. When we run a simulation, the individual behaviour of each cell contributes to the evolution of the system that is visualized by the collective behaviour. Possibly, one may average over several simulations to get insight of the response of the model to different setup of parameters and conditions. According to the remark on the crowding effect, we assume that cells can proliferate only if in their neighborhood some lattice sites are vacant. It is also assumed that dying cells leave immediately blank the site they occupied. In the simplest version that we present here cells are supposed to be immobile, but also a random motion (e.g., diffusion) or drift can be incorporated in the model. Different definitions of "neighbourhood" are possible: for the 2-D lattice Von Neumann's or Moore's neighbourhood can be used (4 orthogonal neighbours - north, south, east, west-or 8 neighbours-adding northwest, northeast, southwest, southeast-respectively). But larger neighbourhood can also be used, such as squares of $5 \times 5$ lattice sites centered in the considered cell. In the simulation we present here, each site in the lattice grid can be in one of three different states:

- vacant sites (white),

- sites occupied by CSC (black),

- sites occupied by CC (red).

Starting from a given situation at time $t_{k}$ each CC red) has a probability $\mu$ of undergoing apoptosis (becoming white). Then, the surviving cells have a probability $\rho$ of being potentially replicant. But, in this case, mitosis is only possible if there are vacant (white) sites in the chosen neighbourhood; if this situation occurs a daughter $\mathrm{CC}$ appears and one of these white sites becomes red. The situation with CSC is similar, but with two differences: (1) $\mu=0$, and (2) if mitosis occurs a daughter CSC appears with probability $\delta$, while the unsymmetrical proliferation (i.e., the appearance of new born $\mathrm{CC}$ ) has probability $1-\delta$. The replication probability $\rho$ can be different for the two classes of cells ( $\rho_{u}$ for CSC, $\rho_{v}$ for CC) and could be assumed to be age-dependent, i.e., decreasing for each mitosis undergone. We will neglect this latter fact and assume that the replication potential is age-independent. We also neglect the possibility of diffusion and movement of the cells, as considered e.g. in Betteridge et al. (2006), Enderling et al. (2009) and Hillen et al. (2013). When the simulation stops before the growing tumour reaches the boundary of the grid, as in the simulations displayed in the sequel, it is not necessary to specify boundary conditions. In other cases the boundary of the grid (not necessarily rectangular) can be identified with a physical boundary beyond which no tumour cell can appear; this means that a form of no-flux boundary conditions are applied and this modifies the form of neighbourhood for the cells close to the boundary. Finally, to mimic an unbounded medium, it can be convenient to impose periodicity, i.e. the boundary "wraps" on itself so that cells of each side of the square interact with cells of the opposite side.

2 For an application to a social problem see Meacci et al. (2012) and Meacci (2015). 


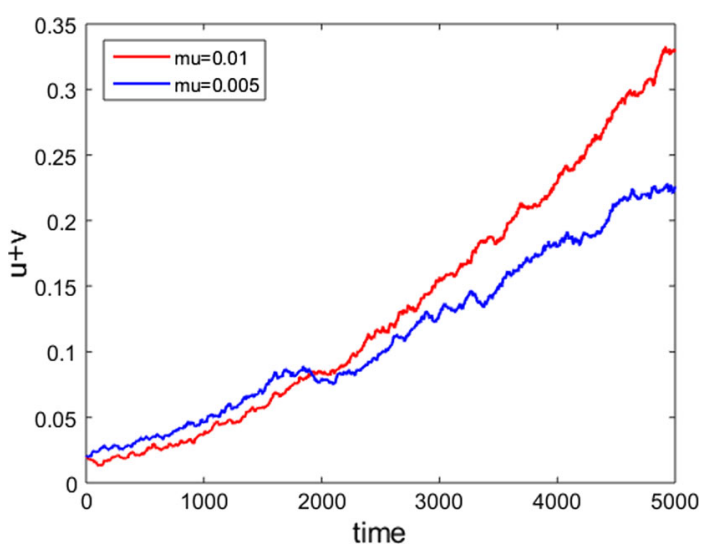

Fig. 1 Fraction of tumour cells (CC plus CSC) as function of time for different mortalities

In Fig. 1 (and later in Figs. 4, 5, 6) we display the evolution with respect to time of the fraction of tumour cells, i.e., the number of lattice sites occupied by tumour cells divided by the total number of the lattice sites (in our case 2500). The Figure shows the results of two simulations corresponding to different mortality of CC. In particular, the two cases considered are with $\mu_{1}=0.005$ (blue line) and $\mu_{2}=0.01$ (red line). The parameters of this simulation are $\delta=0.2, \rho_{u}=0.01$ and $\rho_{v}=0.005$. In Figs. 2 and 3 we show the screenshots correspondent to the two simulations. The results of these simulations show clearly that the model exhibits the tumour paradox. More precisely, a higher number of $\mathrm{CC}$ (case $\mu=0.005$ ) inhibits the growth of CSC and thus of the whole tumour. To conclude this section we consider the case in which, at a prescribed time, a treatment takes place such that all the $\mathrm{CC}$ are destroyed. Displaying the fraction of total number of tumour cells as function of time in the case with treatment and without treatment we see that the treatment produces instantly a reduction of the volume of the tumour but in the long run it does not cause a lower growth of the tumour (see Fig. 4).

We conclude this Section with the following remark. If the initial situation in the CA simulation only consists of a set of red sites, black sites will never appear since CSC can only be generated by stem cells. Thus, this case would formally describe tumours in which stem cells are not present. But in this case the intrinsic approximations introduced in the modelling (in particular age-independence) lead to an oversimplified description in which the final steady state will consist either in a configuration with all white sites (if $\mu>\rho$ ) or in a fixed fraction $(<100 \%)$ of red sites and the tumour paradox does not appear. Therefore, our models only make sense if the initial fraction of CSC is positive.

\section{Mean field approximation}

Consider the situation described in the previous section with no flux boundary conditions and define $u\left(t_{k}\right)$ the fraction of the CSC, i.e., the number of the lattice sites occupied by CSC cells at time $t_{k}$, divided by the total number of sites. Similarly, $v\left(t_{k}\right)$ will represent the fraction of CC at time $t_{k}$. To obtain a mean field approximation, one formally considers [as it was done in Borsi et al. (2015)] that the neighbourhood of each cell is the entire grid, so that the fraction, at time $t_{k}$, of the vacant sites where a new cell can appear is $1-u\left(t_{k}\right)-v\left(t_{k}\right)$. 

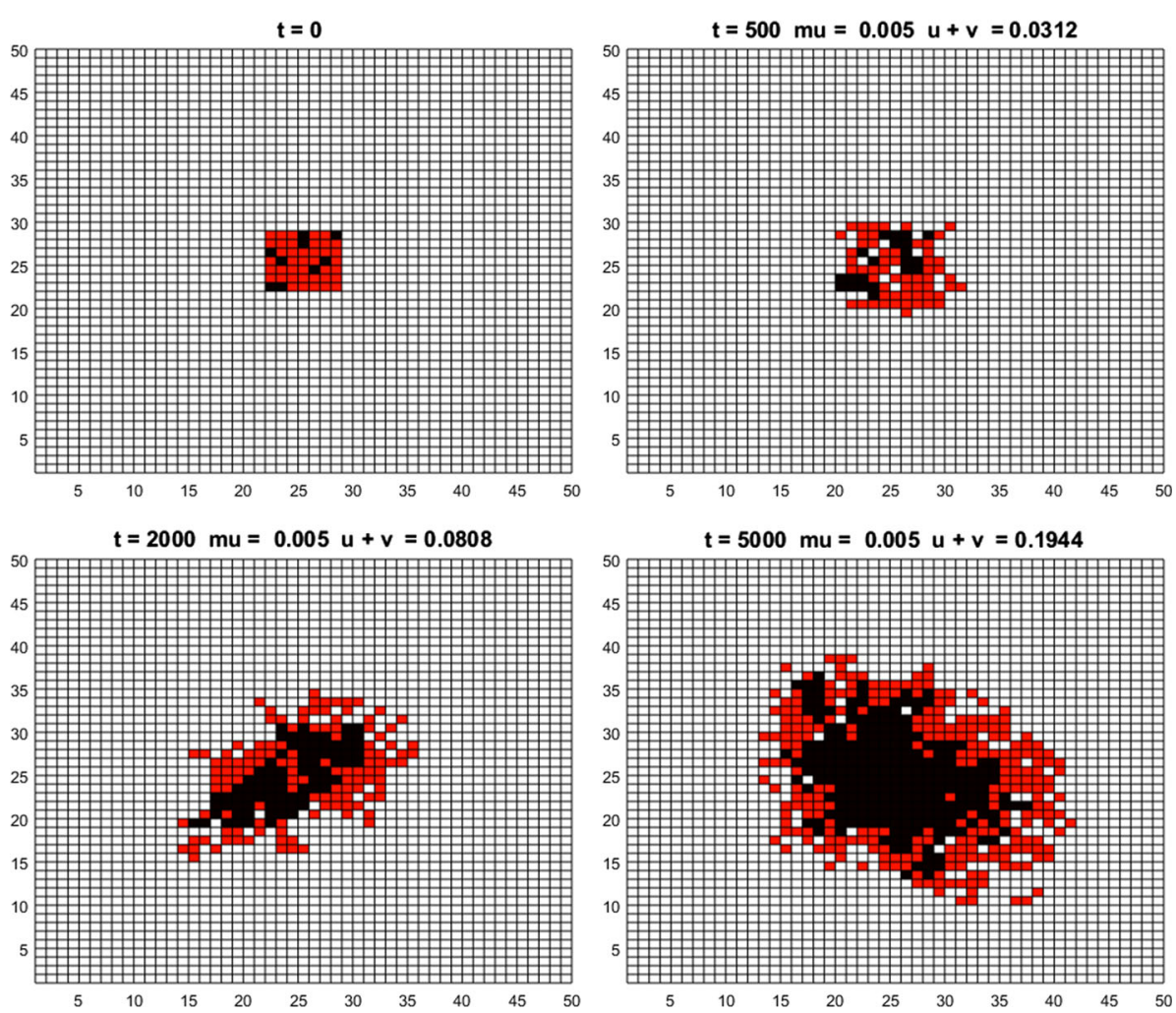

Fig. 2 Distribution of tumour cells CSC (black) and CC (red) for a mortality $\mu_{1}=0.005$ at certain times $(t=0, t=500, t=2000$ and $t=5000)$

Therefore, if we look for a continuous evolution system, we are brought to the following pair of ordinary differential equations: ${ }^{3}$

$$
\begin{gathered}
\frac{\mathrm{d} u}{\mathrm{~d} t}=\rho_{u} \delta u(1-u-v) \\
\frac{\mathrm{d} v}{\mathrm{~d} t}=\rho_{u}(1-\delta) u(1-u-v)+\rho_{v} v(1-u-v)-\mu v
\end{gathered}
$$

According to the remark at the end of Sect. 3, our discussion is confined to tumours with cancer stem cells and thus we assume that $u(0)>0$.

We note that under this assumption the system (1)-(2) has an invariant set $\Sigma=\{(u, v)$ : $u>0, v \geq 0, u+v \leq 1\}$ and has an unique steady state $u=1$ and $v=0$ that is the only global attractor (see Borsi et al. 2015) in the invariant set $\Sigma$. This corresponds to a situation in which, independently of the proliferation and death rate of the two subpopulations, the tumour invades the whole available space and consists of stem cells only. Of course, normalizing time, we have a system with three parameters $\delta, \rho=\rho_{v} / \rho_{u}$ and $\mu^{*}=\mu / \rho_{u}$. To simplify notation we will write $\mu$ instead of $\mu^{*}$ :

\footnotetext{
${ }^{3}$ An interesting generalization of system (1)-(2) is presented in Rodriguez-Brenes et al. (2011, 2015) where the proliferation rate $\rho_{u}$ and the fraction of symmetric mitosis $\delta$ depend on $v$. This produces a sort of feedback regulation of the system that can be broken by phenotypical mutations, thus leading to abnormal cellular growth.
} 

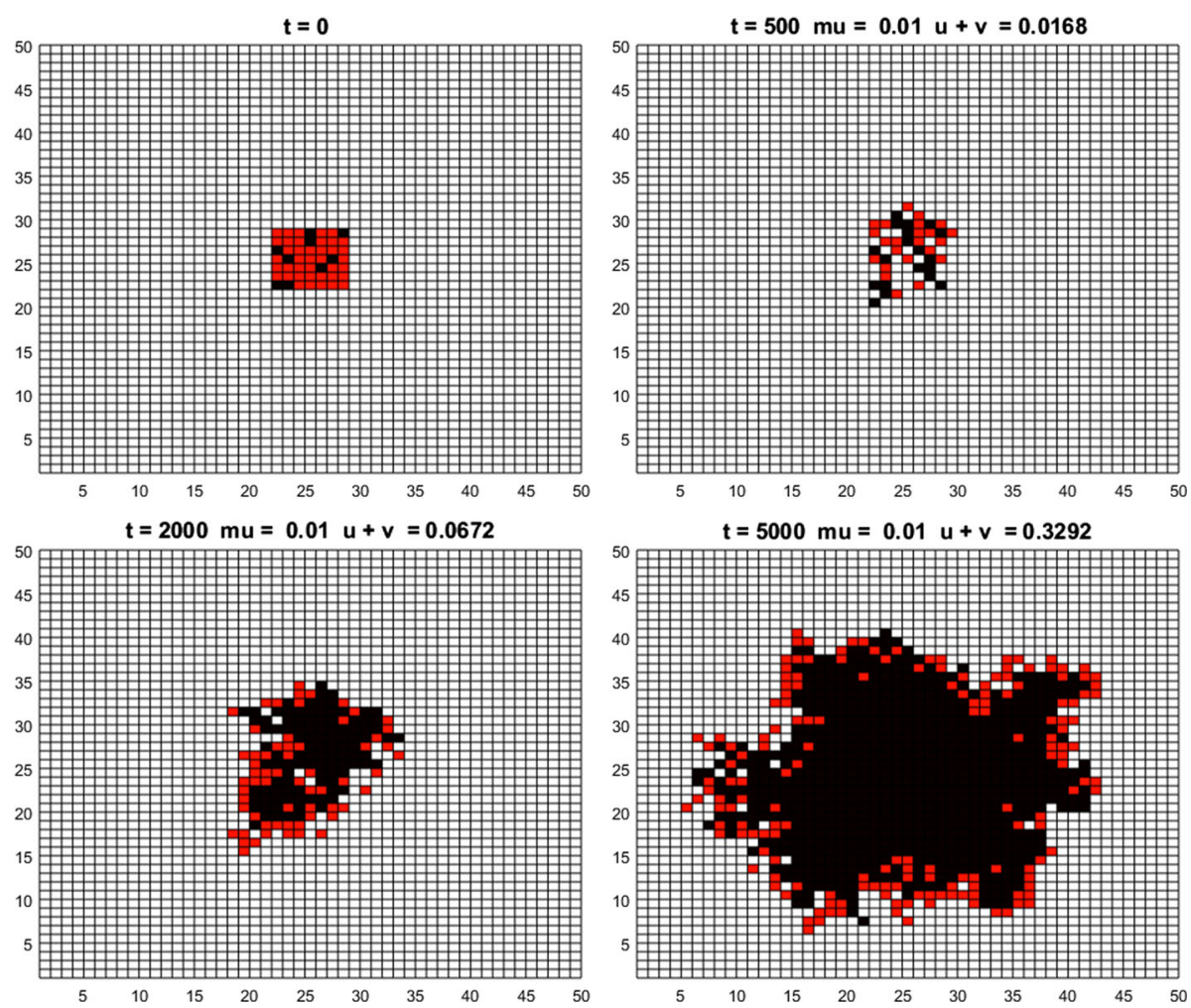

Fig. 3 Distribution of tumour cells CSC (black) and CC (red) for a mortality $\mu_{2}=0.01$ at certain times $(t=0, t=500, t=2000$ and $t=5000)$

$$
\left\{\begin{array}{l}
\dot{u}=\delta u(1-u-v) \\
\dot{v}=(1-\delta) u(1-u-v)+\rho v(1-u-v)-\mu v
\end{array}\right.
$$

Figure 5 shows that the model still exhibits the tumour paradox although this fact is less evident than in the examples displayed in Sect. 3. But this fact can be easily explained: the "crowding" effect is the key factor producing this paradox and in the CA models this effect is taken into account locally, whereas in the mean field approximation the term $(1-u-v)$ just represents the fraction of "empty spaces" irrespectively from their location or distance from the mother cell. To introduce a qualitative correction to this fact the term $1-p(p \equiv u+v)$ has been replaced by $1-p^{n}$ or $\mathrm{e}^{-n p}-\mathrm{e}^{-n}, n>1$ (see Borsi et al. 2015). Also in this mean field approximation we can simulate a treatment that "kills" the $\mathrm{CC}$ at given time. Figure 6 shows an effect that is similar to the one for the CA model presented in Fig. 4 and according to which a sudden decrease of the tumour cells does not provide long-term substantial benefits.

\section{A integro-differential model}

Of course, the mean-field approach only contains global information on the evolution of the tumour but does not take spatial variation into account. In the sequel we present a continuous time model that includes crowding as a local effect, in the sense that a daughter cell can be 


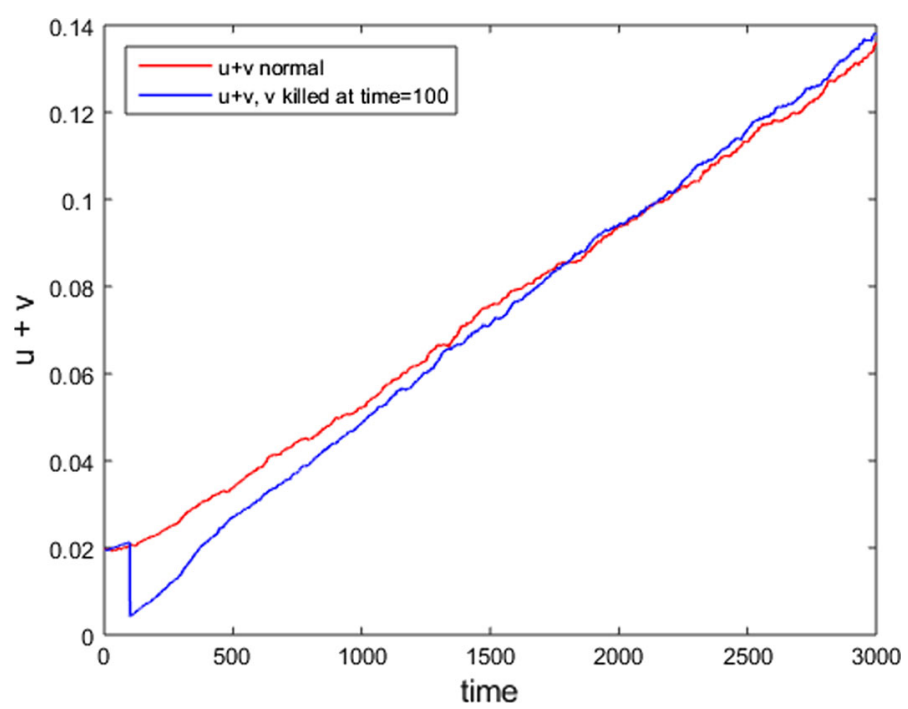

Fig. 4 Fraction of total number of tumour cells (averaged over 20 simulations) as function of time in the case with treatment (blue line) and without treatment (red line). The treatment has the effect of the destruction of the CC cells at the time $t_{d}=100$. Here the setup of parameters is $\mu_{1}=0.005, \delta=0.2, \rho_{u}=0.01$ and $\rho_{v}=0.005$
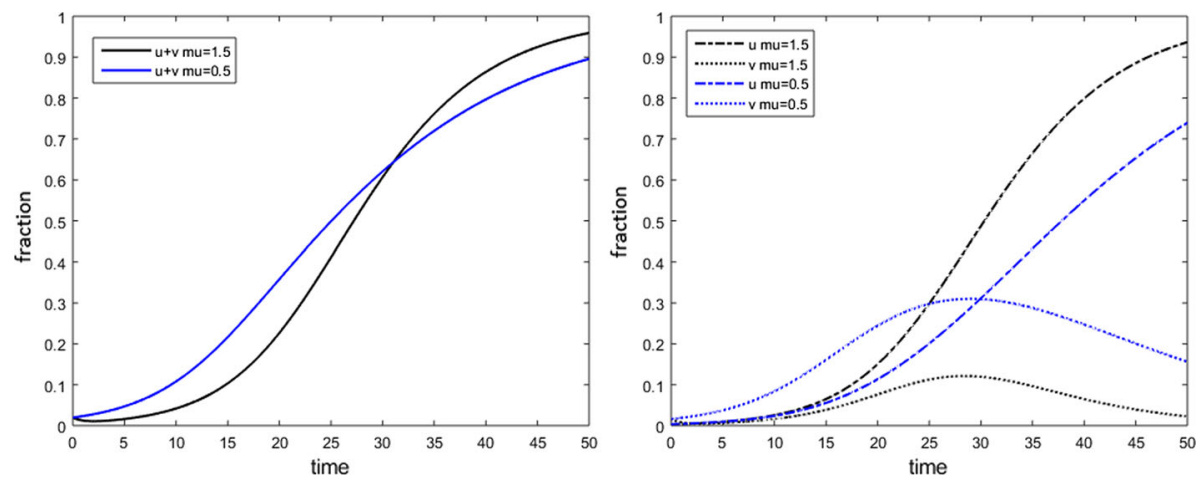

Fig. 5 Fraction of tumour cells $(u+v, u$ and $v$ separately) as function of time. The setup of parameter is $u_{0}=0.016$ and $v_{0}=0.0036$ (initial conditions, as for CA simulations), $\delta=0.2, \rho=0.5$ for the two cases $\mu_{1}=1.5$ and $\mu_{2}=0.5$

generated only if there is space available in a neighbourhood of its progenitor. The radius of this neighborhood is also depending on the possibility that cells are displaced to allow a mitosis to take place. Thus the birth rate of CC's at location $\underline{x}$ originating by CC mother cells can be expressed as

$$
\rho_{v} \int_{\Omega} K(|\underline{x}-\underline{y}|) F((u(\underline{x}, t)+v(\underline{x}, t) \mid) v(\underline{y}, t)) \mathrm{d} y
$$

where $\Omega$ is the domain where the phenomenon takes place ( $\mathbb{R}^{3}$ for simplicity), $K$ is a positive decreasing function whose integral over $\mathbb{R}^{3}$ equals 1 and $F$ is a positive decreasing function such that $F(0)=1, F(1)=0$. In other words, $F$ takes into account the crowding and $K$ is a 


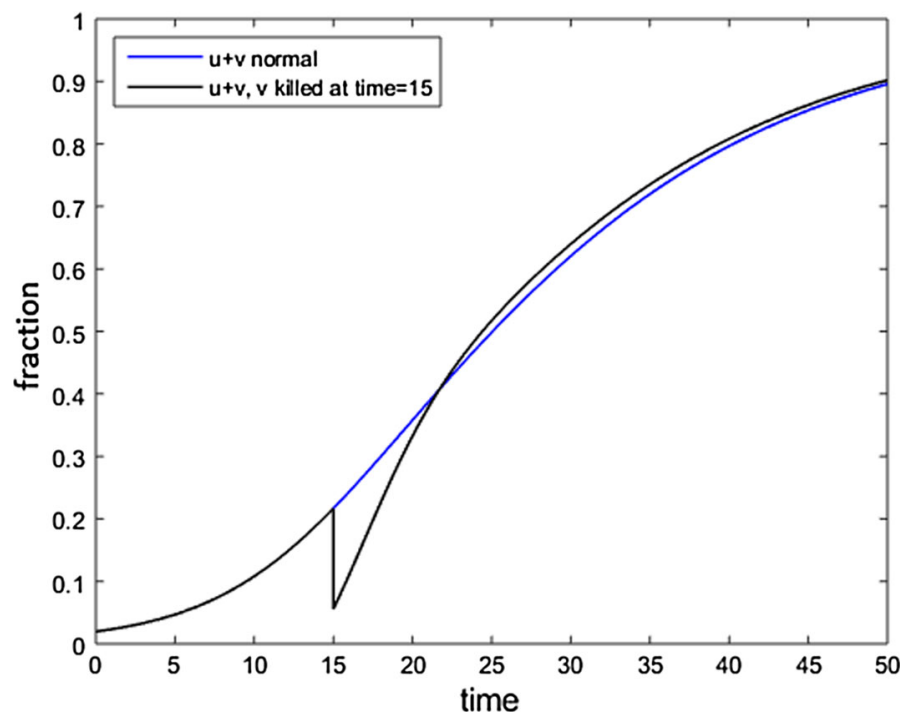

Fig. 6 Fraction of total number of tumour cells as function of time in the case with treatment (black line) and without treatment (blue line). The treatment has the effect of the destruction of the CC cells at the time $t_{d}=15$. Here the setup of parameters is $\rho=0.5, \mu=0.5$ and $\delta=0.2$

kernel indicating "how far" the proliferation capability of a cell can have an effect. Therefore, the system that has to be considered is the following:

$$
\begin{aligned}
\frac{\partial u(\underline{x}, t)}{\partial t}= & \rho_{u} \delta \int_{\Omega} K_{u}(|\underline{x}-\underline{y}|) F((p(\underline{x}, t)) u(\underline{y}, t) \mathrm{d} y \\
\frac{\partial v \underline{x}, t)}{\partial t}= & \rho_{u}(1-\delta) \int_{\Omega} K_{u}(|\underline{x}-\underline{y}|) F((p(\underline{x}, t)) u(\underline{y}, t) \mathrm{d} y \\
& +\rho_{v} \int_{\Omega} K_{v}(|\underline{x}-\underline{y}|) F((p(\underline{x}, t)) v(\underline{y}, t) \mathrm{d} y \\
& -\mu v(\underline{x}, t)
\end{aligned}
$$

where we put

$$
p(\underline{x}, t)=u(\underline{x}, t)+v(\underline{x}, t)
$$

and we assumed, for sake of generality, that the kernels could be different for CSC and CC. Again, we can assume

$$
F((p(\underline{x}, t))=1-p(\underline{x}, t)
$$

and typically

$$
K_{u}(|\underline{x}-\underline{y}|)=\frac{1}{\sqrt{\pi} \sigma_{u}} \exp \left(-\frac{(x-y)^{2}}{\sigma_{u}^{2}}\right) .
$$

In Borsi et al. (2015) it is shown (see also Maddalena 2014) that the model is mathematically well-posed (existence, uniqueness, continuous dependence on the data, boundedness of the solutions) and some numerical simulations are shown that provide evidence of the following basic facts: (1) if the mortality rate of $\mathrm{CC}$ is higher, then the tumour is more aggressive and (2) incomplete treatment of cancers can enhance their growth. Indeed CSC are less (or not) sensitive to treatments and hence the stress applied to the tumour acts in favour of a 

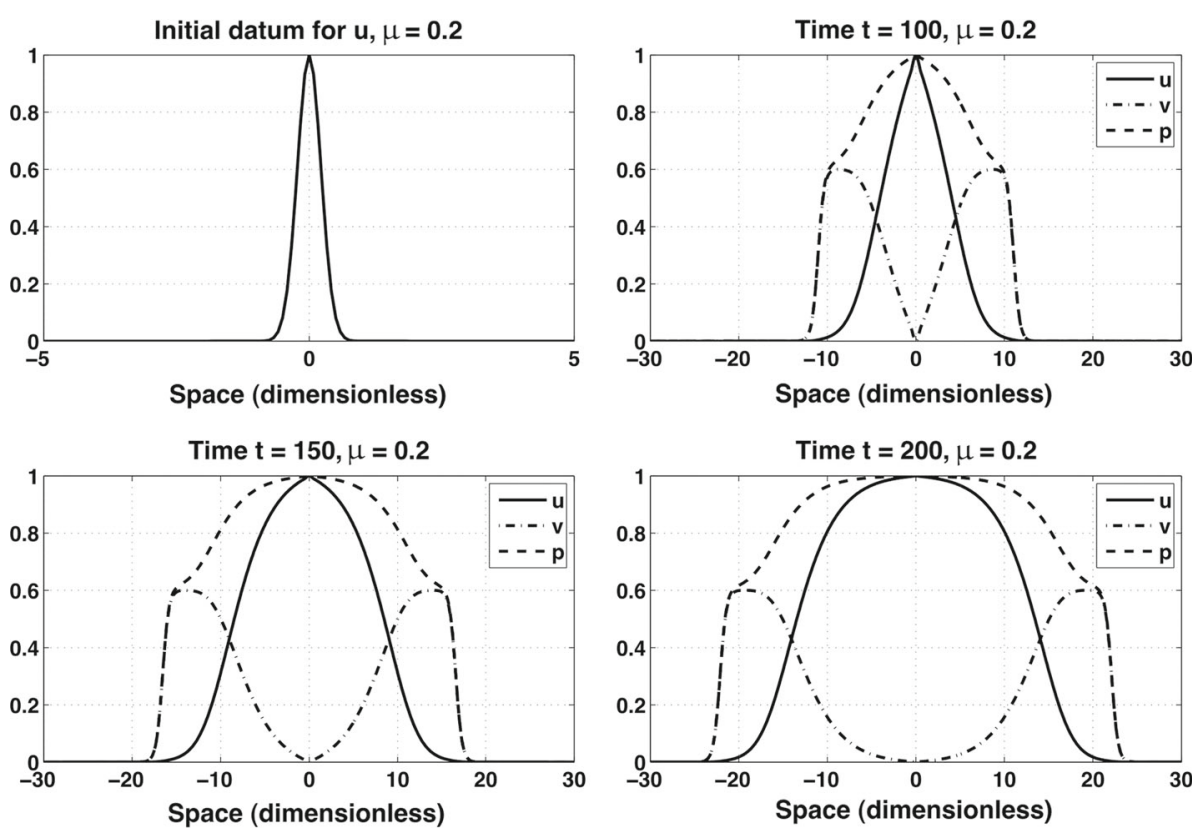

Fig. 7 Plot of $u, v, p=u+v$ at selected time instants, with initial conditions 12 and parameters from 10 and 11. Case $\mu=0.2$

selection of stem cells, thus enhancing the fitness of tumour and its growth. For simplicity, numerical simulations are confined to one-dimensional situation. The choice of parameters is the following

$$
\rho_{u}=1 \quad \rho_{v}=0.5 \quad \delta=0.2
$$

and the kernels are taken as Gaussian curves with

$$
\sigma_{u}=0.5 \quad \sigma_{v}=0.1
$$

The initial conditions are the following

$$
u(x, 0)=\mathrm{e}^{-10 x^{2}} \quad v(x, 0)=0 .
$$

The plots of Figs. 7 and 8 correspond to two different values of the mortality of the CC. In Fig. $7 \mu=0.2$ and the invasion of the tumour is clearly dominated by CC while CSC are in the centre of the cancer. In Fig. $8(\mu=2)$ we see that the progression of the tumour is dominated by CSC. Finally, Fig. 9 displays for two values of $\mu$ the time evolution of the total tumour mass

$$
\Pi(t)=\int_{-\infty}^{\infty} p(x, t) \mathrm{d} x
$$

and the progression of the level line $p=0.8$. Indeed, the latter gives an idea of the motion of the boundary of the tumour and we can take its slope as a measure of the invasion speed of the tumour. Figure 9 (left) shows that tumours with $\mu=2$ have a total mass that, after some time, exceeds the mass of tumours with $\mu=0.2$. The slope of the curves in Fig. 9 (right) shows that tumours with higher mortality of CC have an higher invasion speed. The tumour paradox is evident. 

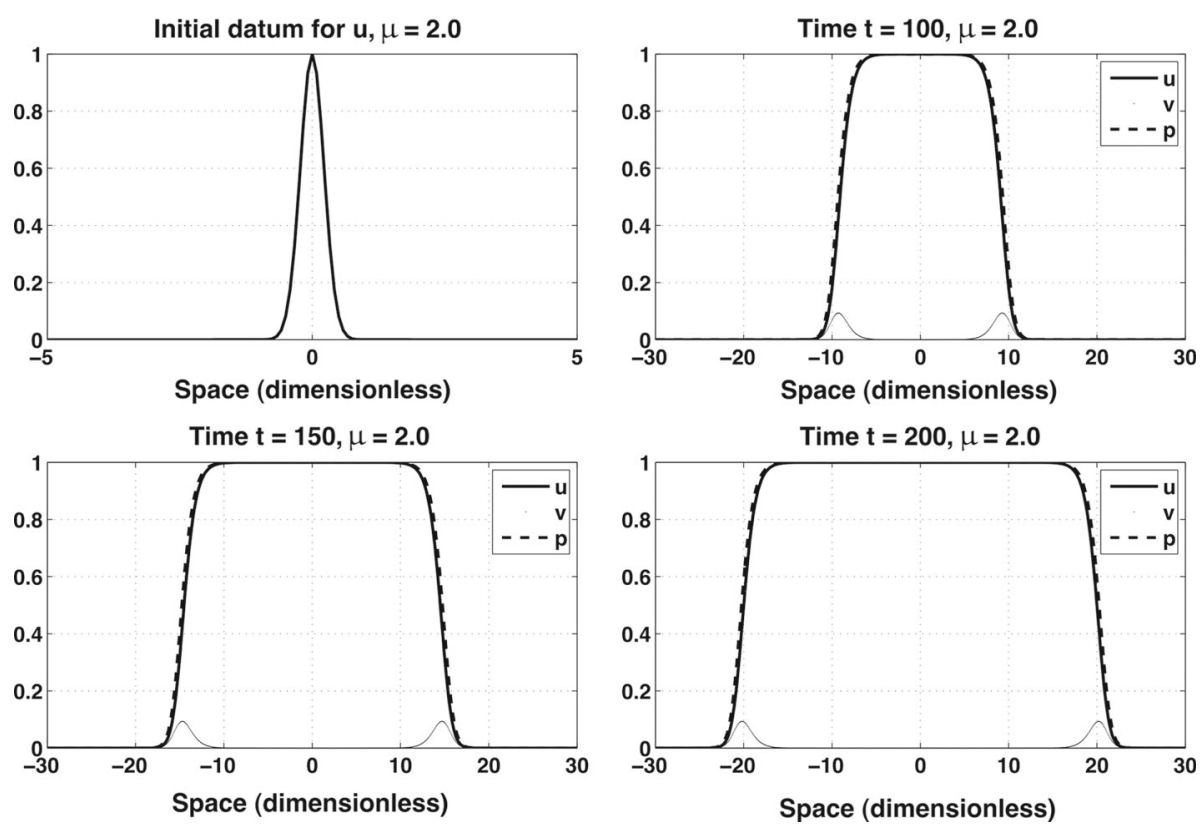

Fig. 8 Plot of $u, v, p=u+v$ at selected time instants, with initial conditions 12 and parameters from 10 and 11. Case $\mu=2$
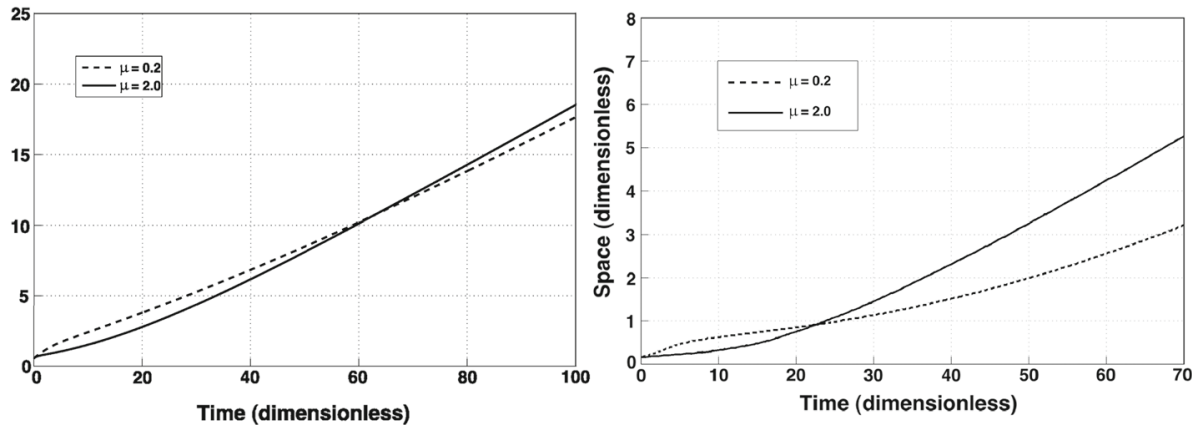

Fig. 9 Left: time evolution of total population $\Pi(t)$, for $\mu=0.2$ (dotted line) and $\mu=2.0$ (solid line). Right: time evolution of the level line $p=0.8$, for $\mu=0.2$ and $\mu=2.0$

The model can also be used to visualize the effect of treatment. Take $\mu=0.2$ and assume that at $t=50$ we apply a radiation treatment that destroys a fraction $\phi$ of the CC and has no effect on CSC. Figure 10 displays $p$ at time $t=50$ (just after the treatment) and $t=100$ and the comparison with the case of no treatment. In Fig. 11 the total tumour mass and the rate of invasion in the two cases are compared. A more precise way of taking into account the effect of treatment should also include (as in Jackson and Byrne 2000) the diffusion of the drug through the extracellular matrix. Here, for sake of simplicity, we consider that the treatment has an instantaneous effect everywhere. 

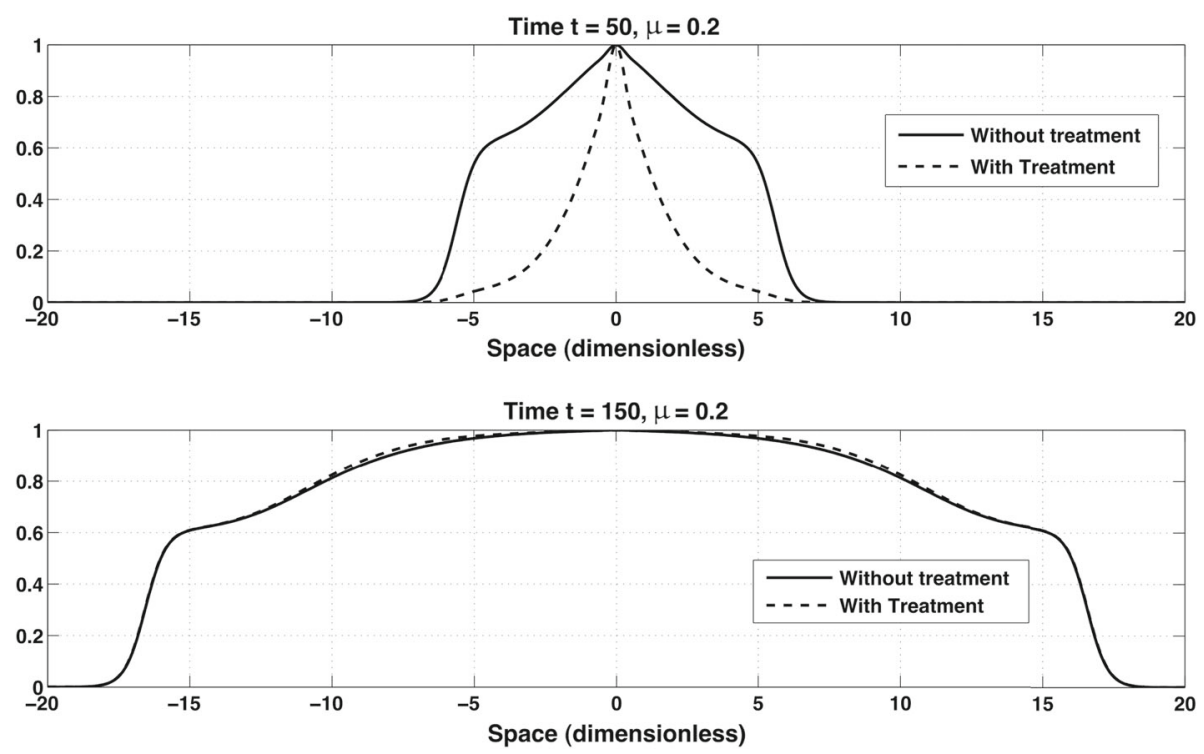

Fig. 10 Plot of $p$ at selected time instants in case of radiation treatment at $t=50$. Case $\mu=0.2$
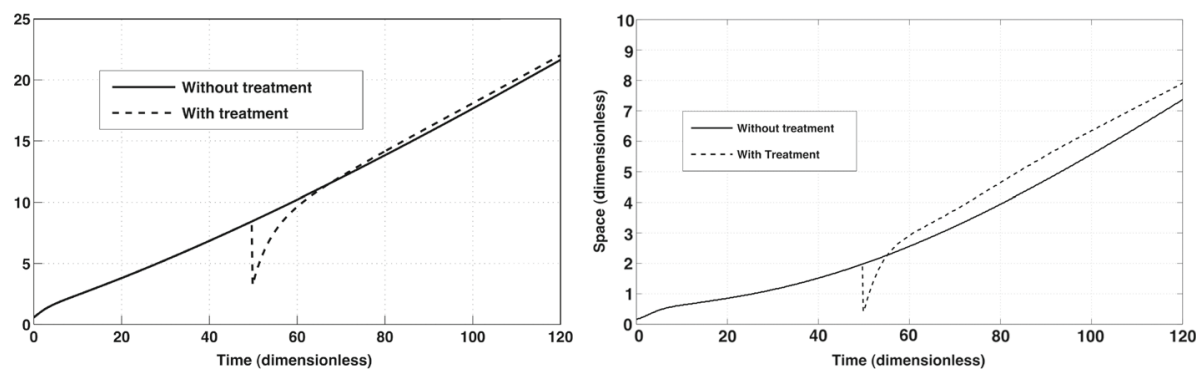

Fig. 11 Left: time evolution of total population $\Pi(t)$, for $\phi=0.95$ and $\mu=0.2$. Right: time evolution of the level line $p=0.8$, for $\phi=0.95$ and $\mu=0.2$

\section{A reaction-diffusion model}

Consider the integral appearing in Eq. (5) in the one-dimensional case $(\Omega \equiv(-\infty,+\infty))$ and approximate $u(y, t)$ till the second order as it was done in Fasano et al. (2016)

$$
\begin{aligned}
& \rho_{v} \int_{-\infty}^{+\infty} K(|x-y|) F((p(x, t)) v(y, t) \mathrm{d} y \\
& \simeq \rho_{v} F\left((p(x, t)) \int_{-\infty}^{+\infty} K(|x-y|)\left[v(x, t)+(y-x) v_{x}(x, t)+(y-x)^{2} \frac{v_{x x}(x, t)}{2}\right] \mathrm{d} y\right. \\
& \simeq \rho_{v} F\left((p(x, t))\left[A v(x, t)+B v_{x x}(x, t)\right]\right.
\end{aligned}
$$



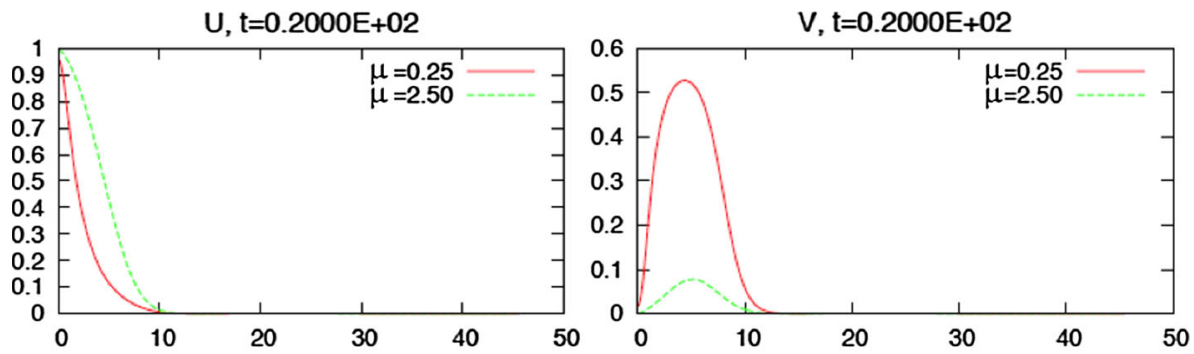

$\mathrm{U}+\mathrm{V}, \mathrm{t}=0.2000 \mathrm{E}+02$

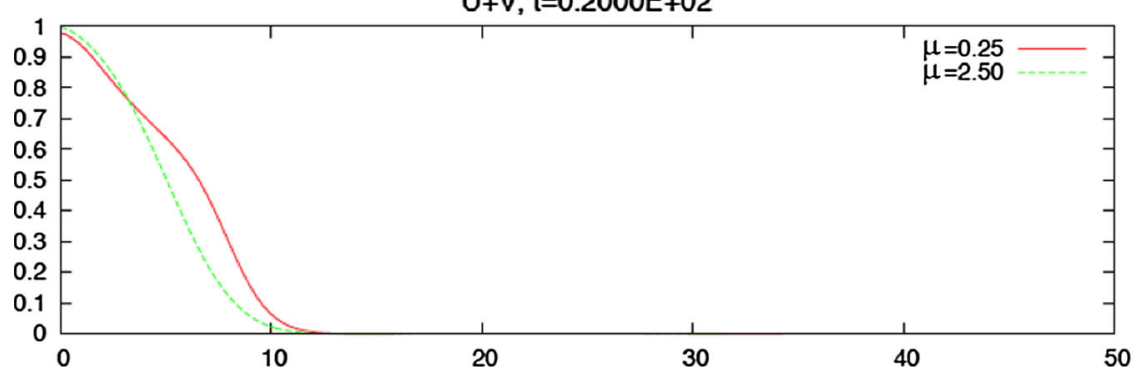

Fig. 12 Plots for $u$, for $v$ and for $p=u+v$ at the time $t=20$

where $A$ is the integral of $K$ and $B$ is half of its second order momentum (the first order momentum vanishes since $K$ is symmetric). In particular, if $K$ is a Gaussian curve

$$
A=1 \quad B=\frac{\sigma_{v}^{2}}{4} .
$$

By this approximation (see (Capasso and Serio 1978)), we transform system (5)-(6) in the following

$$
\begin{gathered}
\frac{\partial u}{\partial t}=\rho_{u} \delta F(p)\left[u+\frac{\sigma_{u}^{2}}{4} u_{x x}\right] \\
\frac{\partial v}{\partial t}=\rho_{u}(1-\delta) F(p)\left[u+\frac{\sigma_{u}^{2}}{4} u_{x x}\right]+\rho_{v} F(p)\left[v+\frac{\sigma_{v}^{2}}{4} v_{x x}\right]-\mu v .
\end{gathered}
$$

System (16)-(17) is a classical reaction-diffusion model (by the way an additional diffusion effect can be taken into account if it is present in the original setting of the problem). In Fasano et al. (2016) a proof is given of the local existence for problem (16)-(17) supplemented with smooth initial data and usual vanishing conditions at $\pm \infty$. Numerical simulations give results quite similar to the ones displayed in the previous section. Using the same setting of parameters and performing numerical simulations with $\mu=0.25$ and $\mu=2.50$ we have the following plots for $u$, for $v$ and for $p=u+v$ at the time $t=20$ (Fig. 12), $t=30$ (Fig. 13). Only the part of the plot for $x>0$ is displayed. Figure 14 shows the time evolution of $\Pi$.

\section{Conclusions}

We have presented four classes of mathematical models to describe the growth of a tumour characterized by the presence of cancer stem cells (CSC). The simpler model averages over the space variables and represents a sort of mean field approximation to the phenomenon 

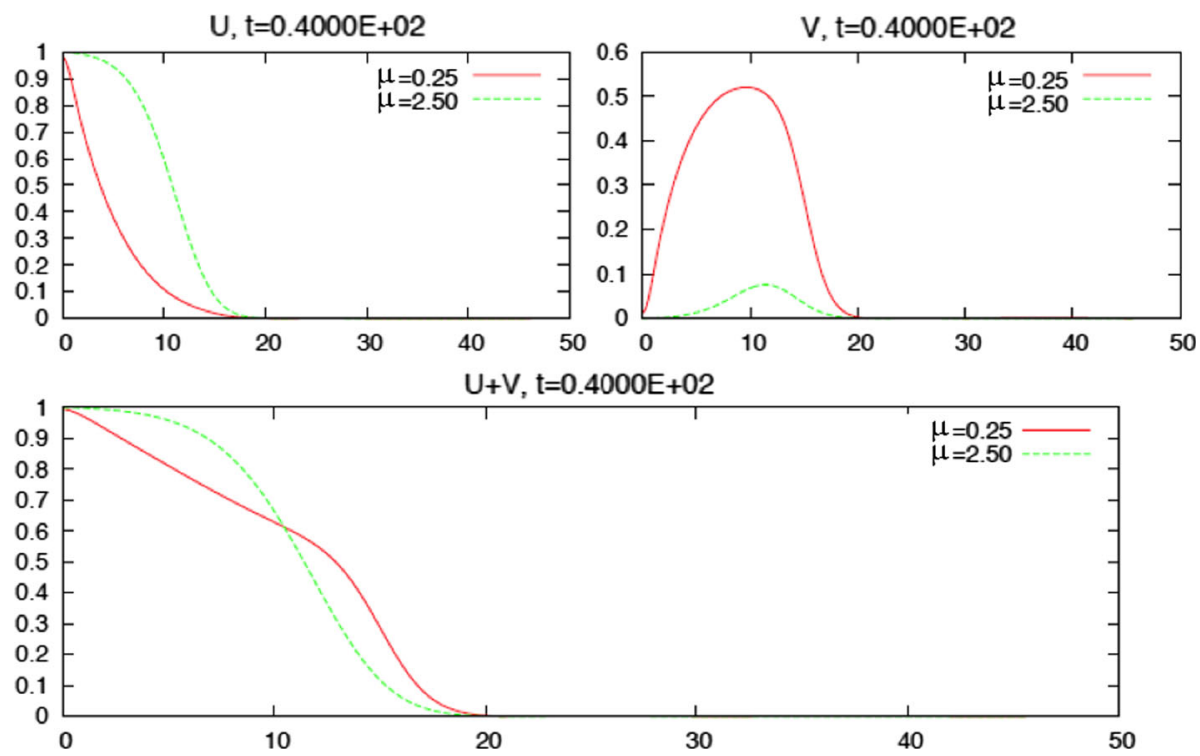

Fig. 13 Plots for $u$, for $v$ and for $p=u+v$ at the time $t=40$

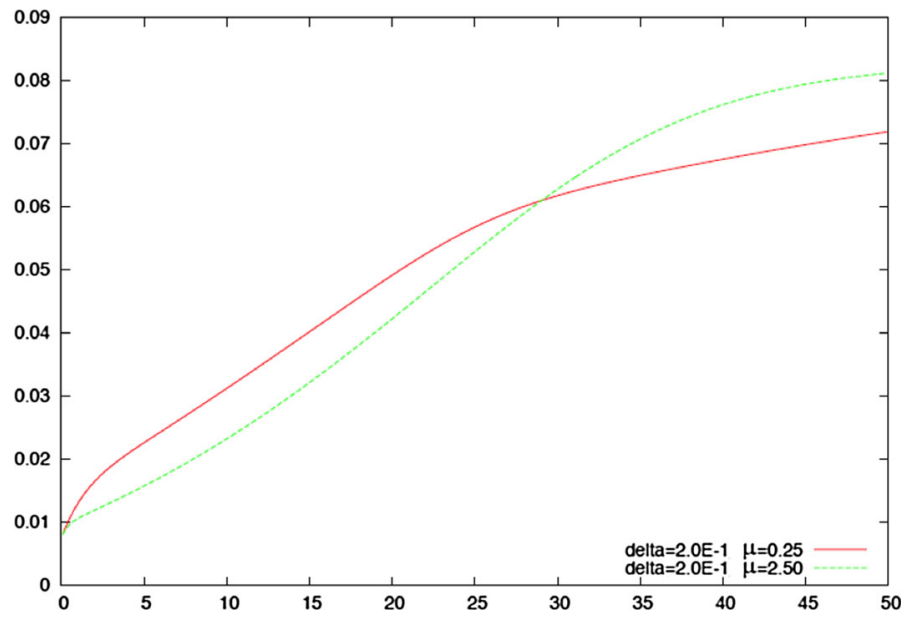

Fig. 14 Time evolution of $\Pi(t)$

thus resulting in a system of ordinary differential equations for the time evolution of the total number of CSC and of ordinary cancer cells (CC). Notwithstanding this (and other) drastic simplifications, our numerical simulations provide evidence of the basic features that characterize tumours with CSC, in particular the so-called "tumour paradox": if the mortality of the $\mathrm{CC}$ is increased the growth of the tumour is accelerated. If space dependence is taken into account, the mathematical aspects of the problem appear to be more complicated. We presented a model based on integro-differential equations and subsequently we have shown how this model (under suitable assumptions) can be transformed in a system of parabolic PDE wth reaction diffusion terms. In these cases, numerical simulations have been performed 
in a one-dimensional setting and show how the radius of the tumour grows with time. Also in this case the effect of "tumour paradox" is present. Simulations with cellular automata have also been performed. In a two-dimensional grid three categories of sites are presents: sites where no cells are present, sites occupied by CSC and sites occupied by CC. The simulations show how the tumour grows, without any prescribed symmetry, and how the two families of cells proliferate. As in the cases described above, the "crowding" effect of the cells appear to be responsible for the occurrence of the tumour paradox. As a consequence, a chemical or radiative treatment that destroys $\mathrm{CC}$ may have the effect of increasing, instead of controlling, the speed at which tumoral cells invade the surrounding space.

As we said, the models do not provide quantitative results but a conceptual framework to interpret experimental data. Simulations with parameters estimated by "in vitro" experiments could just give hints on the strategy of treatment (frequency and intensity of applications) and suggestions on situations to be avoided. Summing up, this paper shows that the tumour paradox can be explained on the basis of a few basic assumptions on the mechanisms of cell proliferation and that the phenomenon could be put in evidence in different classes of mathematical models, considering, therefore, a complete multi-approach point of view.

Acknowledgements Luca Meacci acknowledges with appreciation the foundation CAPES (Coordenação de Aperfeiçoamento de Pessoal de Nível Superior) of the Ministry of Education of Federal Republic of Brazil for receiving economic support within the funding program of academic excellence PROEX (Programa de Excelência Acadêmica). Mario Primicerio acknowledges with appreciation the support of GNFM (Gruppo Nazionale di Fisica Matematica) of the Istituto Superiore di Alta Matematica, Rome. The authors also would like to thank the editor and the reviewers for their detailed comments and efforts to improve the manuscript.

\section{References}

Al-Hajj M, Wicha MS, Benito-Hernandez A, Morrison SJ, Clarke MF (2003) Prospective identification of tumorigenic breast cancer cells. Proc Nat Acad Sci 100(7):3983-3988

Anderson AR, Quaranta V (2008) Integrative mathematical oncology. Nat Rev Cancer 8(3):227

Betteridge R, Owen MR, Byrne HM, Alarcón T, Maini PK (2006) The impact of cell crowding and active cell movement on vascular tumour growth. Netw Heterog Media 1(4):515-535

Borsi I, Fasano A, Primicerio M, Hillen T (2015) A non-local model for cancer stem cells and the tumour growth paradox. Math Med Biol 34(1):59-75

Capasso V, Serio G (1978) A generalization of the kermack-mckendrick deterministic epidemic model. Math Biosci 42(1):43-61

Chopard B, Droz M (1998) Cellular automata modeling of physical systems. Cambridge University Press, Cambridge, pp 122-137

Dingli D, Michor F (2006) Successful therapy must eradicate cancer stem cells. Stem Cells 24(12):2603-2610

Dingli D, Traulsen A, Pacheco JM (2007) Compartmental architecture and dynamics of hematopoiesis. PLoS One 2(4):e345

Dittmar T, Zänker KS (2013) Role of cancer stem cells in cancer biology and therapy. CRC Press, Boca Raton

Enderling H, Anderson AR, Chaplain MA, Beheshti A, Hlatky L, Hahnfeldt P(2009) Paradoxical dependencies of tumor dormancy and progression on basic cell kinetics. Cancer Res 69(22):8814-8821

Fasano A, Mancini A, Primicerio M (2016) Tumours with cancer stem cells: a PDE model. Math Biosci 272:76-80

Ganguly R, Puri I (2006) Mathematical model for the cancer stem cell hypothesis. Cell Prolif 39(1):3-14

Gurova KV, Gudkov AV (2003) Paradoxical role of apoptosis in tumor progression. J Cell Biochem 88(1):128137

Hillen T, Enderling H, Hahnfeldt P (2013) The tumor growth paradox and immune system-mediated selection for cancer stem cells. Bull Math Biol 75(1):161-184

Jackson TL, Byrne HM (2000) A mathematical model to study the effects of drug resistance and vasculature on the response of solid tumors to chemotherapy. Math Biosci 164(1):17-38

Kakarala M, Wicha MS (2008) Implications of the cancer stem-cell hypothesis for breast cancer prevention and therapy. J Clin Oncol 26(17):2813-2820 
Kari J (2005) Theory of cellular automata: a survey. Theoret Comput Sci 334(1):3-33

Kiel MJ, He S, Ashkenazi R, Gentry SN, Teta M, Kushner JA, Jackson TL, Morrison SJ (2007) Haematopoietic stem cells do not asymmetrically segregate chromosomes or retain BRDU. Nature 449(7159):238

Lathia JD, Mack SC, Mulkearns-Hubert EE, Valentim CL, Rich JN (2015) Cancer stem cells in glioblastoma. Genes Dev 29(12):1203-1217

Maddalena L (2014) Analysis of an integro-differential system modeling tumor growth. Appl Math Comput 245:152-157

Marciniak-Czochra A, Stiehl T, Wagner W (2009) Modeling of replicative senescence in hematopoietic development. Aging (Albany NY) 1(8):723

Meacci L (2015) How and how much to invest for fighting cheaters: from an ODE to a cellular automata model. Math Sci 9(3):137-143

Meacci L, Sanz Nuño JC, Primicerio M (2012) Fighting tax evasion: a cellular automata approach. Adv Math Sci Appl 22(2):597-610

Michor F (2008) Mathematical models of cancer stem cells. J Clin Oncol 26(17):2854-2861

Michor F, Hughes TP, Iwasa Y, Branford S, Shah NP, Sawyers CL, Nowak MA (2005) Dynamics of chronic myeloid leukaemia. Nature 435(7046):1267-1270

Rodriguez-Brenes IA, Komarova NL, Wodarz D (2011) Evolutionary dynamics of feedback escape and the development of stem-cell-driven cancers. Proc Nat Acad Sci 108(47):18983-18988

Rodriguez-Brenes IA, Komarova NL, Wodarz D (2014) Cancer-associated mutations in healthy individuals: assessing the risk of carcinogenesis. Cancer Res 74(6):1661-1669. https://doi.org/10.1158/0008-5472

Rodriguez-Brenes IA, Kurtova AV, Lin C, Lee YC, Xiao J, Mims M, Chan KS, Wodarz D (2017) Cellular hierarchy as a determinant of tumor sensitivity to chemotherapy. Cancer Res 77(9):2231-2241. https:// doi.org/10.1158/0008-5472

Rodriguez-Brenes IA, Wodarz D, Komarova NL (2015) Characterizing inhibited tumor growth in stem-celldriven non-spatial cancers. Math Biosci 270:135-141

Solé RV, Rodríguez-Caso C, Deisboeck TS, Saldana J (2008) Cancer stem cells as the engine of unstable tumor progression. J Theor Biol 253(4):629-637

Stiehl T, Marciniak-Czochra A (2012) Mathematical modeling of leukemogenesis and cancer stem cell dynamics. Math Modell Nat Phenome 7(1):166-202

Sun Z, Komarova NL (2015) Stochastic control of proliferation and differentiation in stem cell dynamics. J Math Biol 71(4):883-901

Werner B, Dingli D, Lenaerts T, Pacheco JM, Traulsen A (2011) Dynamics of mutant cells in hierarchical organized tissues. PLoS Comput Biol 7(12):e1002-290

Werner B, Scott JG, Sottoriva A, Anderson AR, Traulsen A, Altrock PM (2016) The cancer stem cell fraction in hierarchically organized tumors can be estimated using mathematical modeling and patient-specific treatment trajectories. Cancer Res 76(7):1705-1713

Wodarz D, Komarova N (2007) Can loss of apoptosis protect against cancer? Trends Genet 23(5):232-237 